\title{
Solução Autônoma de Auditoria de Acordos de Nível de Serviço para Fatiamento de Recursos em Multi-Mercados Baseada em Blockchain
}

\author{
Jeffson C. Sousa ${ }^{1}$, Billy A. Pinheiro ${ }^{2}$, Elder Bruno Evaristo C. ${ }^{1}$, \\ Antônio J. G. Abelém ${ }^{1}$ \\ ${ }^{1}$ Grupo de Pesquisa em Redes de Computadores e Comunicação Multimídia (GERCOM) \\ Universidade Federal do Pará (UFPA) \\ Caixa Postal 470 - 66075-110 - Belém - PA - Brazil \\ ${ }^{2}$ Amazônia Blockchain Solutions (Amachains) \\ Parque de Ciência e Tecnologia do Guamá (PCT Guamá), Espaço Empreendedor \\ 66075-750 - Belém - PA - Brazil \\ jeffson.sousadicen.ufpa.br, billy@amachains.com, ebec2012@gmail.com \\ abelem@ufpa.br
}

\begin{abstract}
The Slice as a Service (SlaaS) paradigm offers promising opportunities to support various enterprises and institutions that have been using cloud computing to reduce costs and connect their network across different administrative domains. The Novel Enablers for Cloud Slicing (NECOS) platform presents a distributed market model that dynamically creates network slices in the cloud involving different slice resource providers. Proper selection, negotiation, and monitoring of service level agreement applications across multiple administrative and technology domains are among the key challenges for realizing a distributed marketplace of large network resource providers. This paper proposes an SLA compliance auditing model for blockchain-based network resource slicing for transparent and distributed monitoring using smart contracts.
\end{abstract}

Resumo. O paradigma de Fatias como Serviço (Slice as a Service - SlaaS) oferece oportunidades promissoras de suporte a várias empresas e instituições que vem utilizando a computação em nuvem para reduzir custos e conectar a sua rede em diferentes domínios administrativos. A plataforma Novel Enablers for Cloud Slicing (NECOS) apresenta um modelo de mercado distribuído que cria dinamicamente fatias de rede na nuvem envolvendo diferentes provedores de recursos de fatia. A seleção, negociação e monitoramento adequado das aplicações de acordos de nível de serviço entre vários domínios administrativos e tecnológicos estão entre os principais desafios para a realização de um mercado distribuído de grandes provedores de recursos de rede. Este artigo propõe um modelo de auditoria de conformidade de SLA para fatiamento de recursos de rede baseado em blockchain para monitoramento transparente e distribuído, utilizando contratos inteligentes. 


\section{Introdução}

A evolução de tecnologias baseadas em nuvem ganhou atenção na última década ao fornecer diferentes tipos de serviços ao usuário final, como, software como serviço (Software as a Service - SaaS), infraestrutura como serviço (Infrastructure as a Service - IaaS), e rede como serviço (Network as a Service - NaaS). Tornando-se um modelo de negócios na qual o usuário obtém os serviços on-demand através da internet com várias vantagens como elasticidade, flexibilidade, multi-locatário, escalabilidade, dentre outras. Por esta razão a maioria das empresas, instituições e organizações governamentais estão utilizando os serviços da nuvem [Kumar et al. 2021].

Advento desta evolução e variedade de serviços de nuvem, surge um eminente desafio para que tais serviços sejam fornecidos sob demanda respeitando os acordos de nível de serviço (Service Level Aggrement - SLA) utilizando recursos de computação, conectividade e armazenamento que são gerenciados separadamente. Este desafio impulsiona a criação de soluções como GEN] 1 , que automaticamente agenda e provisiona recursos heterogêneos em rede, e o RESERVOIR ${ }^{2}$, que permite alocação e implantação automática de recursos a medida que estes são solicitados [Farias et al. 2019].

Tais soluções não fornecem uma abstração única com abrangência fim-a-fim dos recursos da nuvem (processamento e armazenamento). Para fornecer tal abstração, o conceito de fatia como serviço (Slice as a Service - SlaaS), oriundo do paradigma da Divisão de Rede na Nuvem (Cloud Network Slicing - CNS), é um dos principais capacitadores para cumprir este requisito. Estes serviços de redes fim-a-fim (end-to-end - E2E) autocontidas devem ser mutuamente isolados uns dos outros e suficientemente flexíveis para acomodar casos de uso simultaneamente relacionados a negócios de diferentes locatários em uma infra-estrutura compartilhada [Maciel et al. 2019].

Uma fatia aproveita tecnologias como SDN (Software Defined Networking) e NFV (Network Functions Virtualization), permitindo construir uma estrutura programável e dinâmica sob demanda [Soenen et al. 2017]. As fatias podem ser compostas por instâncias de Virtualized Network Functions (VNFs), recursos de infra-estrutura (por exemplo, CPUs, roteadores, túneis), e serviços de conectividade que abrangem vários domínios administrativos simultaneamente implantado em diferentes configurações de rede [Swapna et al. 2019].

O conceito de multi-mercado pode ser definido como uma plataforma que reúne os fornecedores de recursos multi-domínio e locatários, com uma visão de comercializar partes de fatias (ou seja, VNFs, armazenamento, largura de banda) de forma eficiente segundo as exigências dos locatários através de um modelo de descoberta de recursos de provisionamento dinâmico de fatias E2E [Swapna et al. 2019].

Este artigo explora a relação entre o gerenciamento de acordos de nível de serviço e problemas de confiança, em um cenário de comercialização de fatias de rede em múltiplos mercados de descoberta de recursos. Para construir uma relação de confiança e transparência no tratamento dos contratos de SLA. Este artigo tem como objetivo propor um modelo de auditoria de conformidade de SLA para fatiamento de recursos de rede baseado em blockchain para monitoramento transparente e distribuído, utilizando contratos

\footnotetext{
1 https: //www.geni.net/

2https://www.reservoir-fp7.eu/
} 
inteligentes, para minimizar os problemas de violação entre as entidades envolvidas no processo.

Na seção 2 são apresentados desafios para o processo de auditoria de acordos de nível de serviço, baseado nos conceitos de CNS e SlaaS. Na seção 3 são descritos os trabalhos relacionados a esta proposta. Na seção 4 é representado o modelo de arquitetura distribuída de auditoria de contratos de SLA. A seção 5 mostra um caso de uso da proposta com base no cenário de multimercado da plataforma NECOS.

\section{Desafios para Auditoria em Contratos de Níveis de Serviço}

Um dos maiores desafios do paradigma da computação em nuvem é atender às diversas demandas de consumidores por novos serviços, modificando a forma de implantação de redes de tamanhos fixos para tamanhos variáveis, possibilitando atender aos requisitos de desempenho específicos quanto à latência, escalabilidade, disponibilidade e confiabilidade, impostos por cada caso de uso. Tal questão foi solucionada através da integração dos domínios de rede, computação em nuvem, e de suas operações de gerenciamento. Com isso, permitiu-se uma grande economia, garantindo a capacidade de ampliar a flexibilidade do provisionamento de serviços nessas áreas [Farias et al. 2019]; [Maciel et al. 2019]; [NECOS 2019].

Em um cenário multi-mercado, onde múltiplos provedores de recursos solicitam e fornecem fatias, não há confiança no processo de requisição destas fatias. Isso faz com que processos importantes sejam prejudicados, no que se refere a aplicação de penalidades referentes a violações de SLA e no próprio monitoramento dos serviços nas fatias destes provedores. Dessa forma, a integridade e rastreabilidade da rede é necessária e a combinação de tecnologias de computação em nuvem (CNS, SlaaS, etc) com Blockchain, além de abrir novas oportunidades, pode evitar gargalos e pontos de falhas na rede, por meio da descentralização da blockchain [Seitz et al. 2018].

O projeto Novel Enablers for Cloud Slicing (NECOS) é um exemplo de caso de uso que se baseia nos conceitos de multi-mercado, onde define o termo CNS como sendo um conjunto de componentes de infraestruturas (rede, nuvem, data center), funções de rede, recursos de infraestrutura (ou seja, conectividade, computação e recursos gerenciáveis de armazenamento) e funções de serviço com atributos projetados especificamente para atender às necessidades de uma indústria vertical ou de um serviço [Silva et al. 2018]. Esta seção irá abordar aspectos principais da plataforma NECOS e conceitos de blockchain relevantes para a compreensão da arquitetura.

\subsection{Novel Enablers for Cloud Slicing (NECOS)}

A solução NECOS baseia-se no conceito Lightweight Slice Defined (LSDC), que estende os paradigmas de virtualização e Virtualized Infrastructure Manager (VIM) sob demanda a todos os recursos de rede e banco de dados [Clayman et al. 2018]. O sistema LSDC permite o tempo de execução dinâmico e criação de fatias E2E completas a partir de um conjunto de componentes. Isso representa uma nova abordagem para automatizar o processo de configuração otimizada para nuvem, criando o conceito de fatia de rede em nuvem para todos os recursos dos datacenters federados, enquanto fornece gerenciamento consistente de recursos de computação, conectividade e armazenamento [Farias et al. 2019]. 
Como é possível observar na Figura 1, o processo de identificação dos recursos de software na plataforma NECOS é composto essencialmente por um conjunto de fluxos de trabalho para troca de informações, que incluem solicitações de fatias e recursos, e ofertas destes recursos. Para realizar este processo são necessários os componentes, slice builder (localizado na área esquerda da arquitetura no centro em azul no componente Slice Provider) o slice broker e os slices agents, componentes-chave para o funcionamento do mercado de fatiamento do NECOS, localizados na área direita no componente Resource Marketplace em amarelo [Maciel et al. 2019].

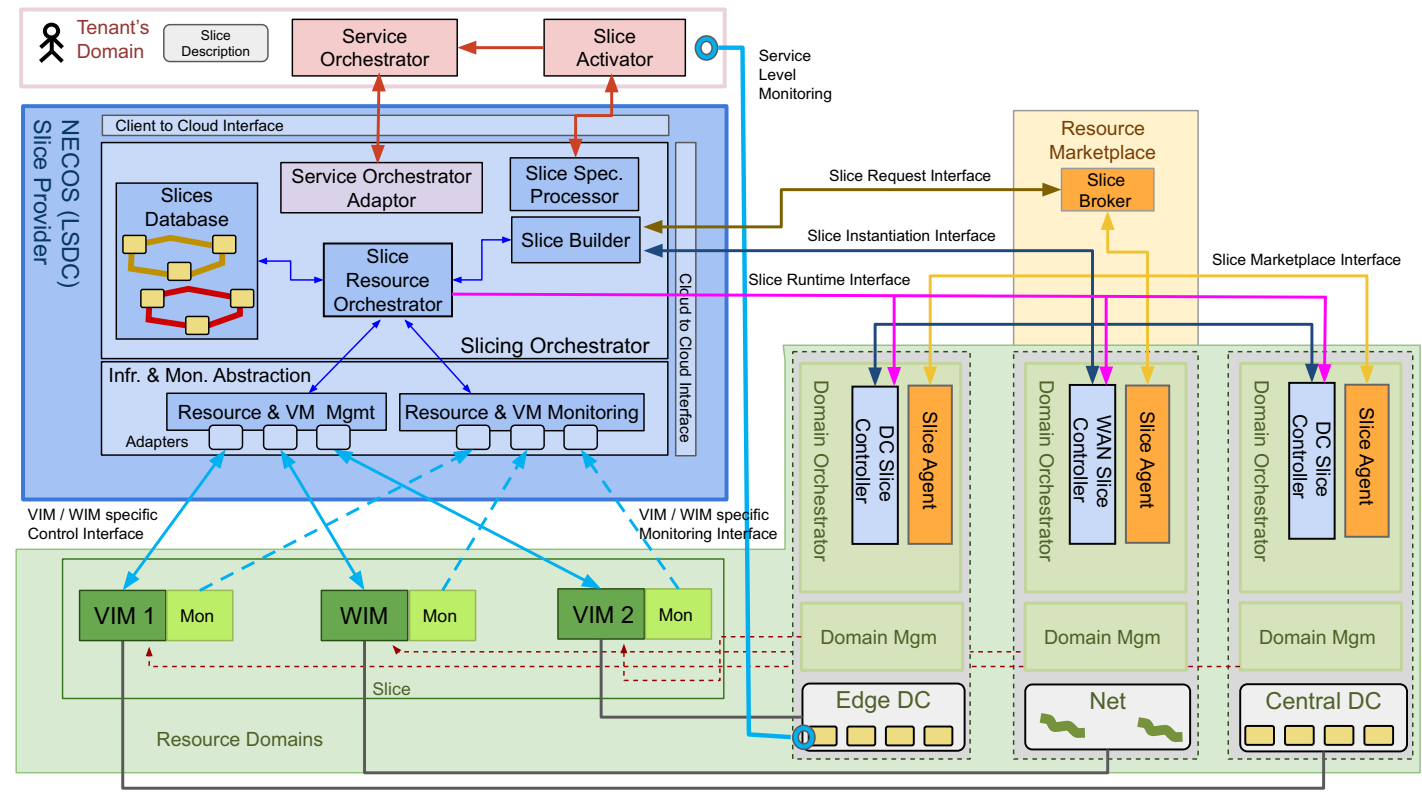

Figura 1. Arquitetura da plataforma [NECOS 2019]

O componente Resource Marketplace tem como principal responsabilidade a descoberta de recursos que irão compor uma fatia. Dessa forma, pode-se afirmar ser um sistema distribuído responsável por localizar partes de fatia adequadas a partir de um conjunto de infraestruturas de nuvem federadas. Logo, possui a função de gerenciar, orquestrar e controlar de forma independente os recursos de nuvem, rede e de hardware [Silva et al. 2018].

O locatário do NECOS, representado na parte superior esquerda da Figura 1, pode ser uma organização que solicita ao subsistema do NECOS Slice Provider a instanciação de recursos heterogêneos, incluindo computação, armazenamento e rede no formulário de fatia de ponta a ponta e executa seu próprio Service Orchestrator para implantar e gerenciar seus próprios serviços nessa fatia da rede alocada. É provável que o locatário tenha uma gama de opções para escolher um provedor de fatias, e isso pode ser baseado em fatores como comércio, localização ou obrigações contratuais.

No NECOS é pressuposto que as tarefas de gerenciamento são impulsionadas pela existência de SLAs entre os locatários e os prestadores, bem como entre os diferentes prestadores. Na arquitetura o detalhamento da especificação de SLA fica fora do âmbito do projeto deixando de fora aspectos legais e econômicos relacionados à violação do SLA. Nela, é explorado uma alternativa que descreve alguns conceitos de elasticidade 
desenvolvidos no projeto e são descritos exemplos de SLAs comuns, sendo o caso do SLO (Service Level Objective). O projeto usa a linguagem de especificação SLO chamada SLO-ML (Service Level Objective Machine Learning) que adota uma sintaxe JSON para representar SLOs [Elhabbash et al. 2019].

Apesar de propor uma solução para atender às diversas demandas de consumidores por novos serviços, o projeto deixa alguns desafios em aberto. Como os locatários que atuam no NECOS são grandes organizações provedoras de recursos, pode haver uma falha deliberada de processos importantes de informações de fluxo.

\subsection{Blockchain}

Blockchain é um mecanismo que permite que as transações sejam verificadas por um grupo de atores não confiáveis. Ele fornece um livro razão distribuído, imutável, transparente, seguro e auditável. A blockchain pode ser consultada de forma aberta e completa, permitindo o acesso a todas as transações ocorridas desde a primeira transação do sistema, podendo ser verificada e conferida por qualquer entidade a qualquer momento. As informações são estruturadas em uma cadeia de blocos, onde cada bloco armazena um conjunto de transações realizadas em um determinado momento. Os blocos são vinculados por uma referência (hash) para o bloco anterior, formando uma corrente encadeada de blocos e fornecendo uma rede distribuída, imutável, transparente segura e auditável [Reyna et al. 2018].

\subsubsection{Contratos Inteligentes}

Uma das principais características de um contrato inteligente é que ele tem uma maneira de fazer cumprir ou executar as cláusulas contratuais por conta própria. Até o surgimento da blockchain, isso era tecnologicamente inviável. A Blockchain acabou sendo ideal para oferecer suporte a contratos inteligentes. Além disso, os contratos inteligentes contribuíram significativamente para o ímpeto da blockchain, esse acoplamento levou a uma segunda geração de blockchain, comumente conhecida como Blockchain 2.0. A combinação de contratos executados automaticamente em um ambiente confiável sem controle centralizado promete mudar a forma como os negócios atuais são feitos [Reyna et al. 2018].

Basicamente, o código do contrato inteligente é armazenado na blockchain e cada contrato é identificado por um endereço único. Para que os usuários operem com ele, basta enviar uma transação para esse endereço. A execução correta do contrato é imposta pelo protocolo de consenso da blockchain. Os contratos inteligentes apresentam um conjunto de vantagens, como redução de custos, velocidade, precisão, eficiência e transparência, que fomentaram o surgimento de muitos novos aplicativos em uma ampla variedade de áreas [Reyna et al. 2018]. O Ethereum foi uma tecnologia blockchain pioneira a incluir contratos inteligentes [Buterin et al. 2014].

\section{Trabalhos Relacionados}

O trabalho de Lieto et al (2018) se baseia no conceito de fatiamento limitando-se a um único mercado de descoberta de recursos, porém não é citado nenhuma proposta de ferramenta de auditoria, ou monitoramento de SLA, distribuída da rede. Os trabalhos de 
Sciancalepore et al (2019) e Habibi et al (2018) referem-se apenas ao SLA, como ferramentas de monitoramento e controle, sem abstração do conceito de auditoria dos acordos de nível de serviço em múltiplos domínios. O único trabalho que faz referência ao paradigma de Fatiamento da Rede é a proposta de Sciancalepore et al (2019) porém sem mencionar nenhuma ferramenta ou arquitetura distribuída para lidar com multi-mercado.

Nos trabalhos de Afraz e Ruffini (2020) e Rebello et al (2019) uma ferramenta distribuída da rede (DLT) é proposta, porém em nenhum dos artigos é tratado o conceito de auditoria de SLA de forma profunda. Apesar de se basearem no modelo de Fatiamento de Rede, nenhum trata a respeito do conceito de multi-mercado para descoberta de recursos em diferentes domínios administrativos.

A proposta de Backman et al (2017) e Valtanen et al (2018) definem uma arquitetura, porém não realizam uma implementação. Elas se atem apenas a teoria, sem mostrar um modelo para fatiamento de rede voltado para multi-mercado. O trabalho de Rosa e Rothenberg (2018) e Swapna et al (2019) têm uma proposta de arquitetura voltada para o modelo conceitual de fatiamento de rede para múltiplos domínios administrativos, os autores discutem o uso de DApps (aplicativos descentralizados) construídos na blockchain para permitir a orquestração de serviços multi-domínio, onde propõe contratos inteligentes a serem usados como SLAs para permitir transparência contra acordos entre domínios administrativos. No entanto, a interpretação do contrato inteligente ainda não foi explorada.

Rathi et al (2020) propõem um esquema de mercado que aborda o gerenciamento do ciclo de vida de VNFs de vários fornecedores habilitando uma blockchain para a coordenação confiável de orquestradores de borda em um cenário multi-domínio que podem fornecer fatias E2E usando recursos adquiridos de vários operadores em um mercado específico. Para analisar o comportamento do sistema de orquestração de borda de múltiplos domínios habilitado para blockchain, o artigo realiza uma simulação experimental de uma rede Hyperledger implementada usando a estrutura Fabric. As solicitações E2E geradas e os dados relativos aos tempos de resposta foram analisados para identificar o comportamento da resposta do sistema. Apesar de abordar o conceito de fatiamento de rede, inclusive utilizando uma blockchain permissionada, os autores não abordam o paradigma de multi-mercado em diferentes domínios administrativos.

Tabela 1. Soluções de Fatiamento de Rede em Múltiplos Domínios

\begin{tabular}{lccc}
\hline Papers & $\begin{array}{c}\text { Cloud Network Slice Multi- } \\
\text { Domain }\end{array}$ & $\begin{array}{c}\text { Audit SLA } \\
\text { Blockchain-Based }\end{array}$ & $\begin{array}{c}\text { Marketplace for } \\
\text { Multidomain }\end{array}$ \\
\hline Afraz and Ruffini 2020 & $\mathrm{X}$ & - & - \\
Habibi et al. 2018 & $\mathrm{X}$ & - & - \\
Lieto et al. 2018 & $\mathrm{X}$ & - & - \\
Sciancalepore et al. 2019 & $\mathrm{X}$ & - & - \\
Rebello et al. 2019 & $\mathrm{X}$ & - & - \\
Backman et al. 2017 & $\mathrm{X}$ & - & - \\
Valtanen et al. 2018 & $\mathrm{X}$ & - & - \\
Rosa and Rothenberg 2018 & $\mathrm{X}$ & - & - \\
Rosa, Rothenberg, Swapna et al. & $\mathrm{X}$ & - & $\mathrm{X}$ \\
2019 & $\mathrm{X}$ & & \\
Rathi et al. 2020 & $\mathrm{X}$ & $\mathrm{X}$ & - \\
Proposta & & $\mathrm{X}$ & $\mathrm{X}$ \\
\hline
\end{tabular}

A implantação futura do $5 \mathrm{G}$ envolverá a administração de vários domínios para fornecer vários serviços 5G (IoT, AR e VR). Essa implantação precisa ser distribuída e au- 
tomatizada (gerenciamento/orquestração) para atender aos SLAs especificados. Portanto, as soluções baseadas em Blockchain são altamente desejáveis para resolver os desafios envolvidos com domínios administrativos não confiáveis, como transações complexas entre domínios, faturamento e SLAs. Com essa premissa, este trabalho tem como proposta inicial dar continuidade direta ao trabalho de Swapna et al (2019), com uma proposta de utilização de uma rede distribuída semelhante à desenvolvida em Rathi et al (2020), mas com o modelo multi-mercado.

\section{Arquitetura da Solução Autônoma de Auditoria de Acordos de Nível de Serviço para Fatiamento de Recursos em Multi-Mercados Baseada em Blockchain}

Cada fatia da rede vem com certas garantias qualitativas, geralmente na forma de um SLA pré-negociado. Uma solução de auditoria de SLA inteligente pode minimizar os problemas de não conformidade entre SLAs em um cenário de fatiamento de rede de vários mercados. Para auxiliar na criação do processo de confiança e transparência, é proposta uma solução distribuída autônoma para o tratamento de métricas de SLA, inseridas em contratos inteligentes de um registro distribuído, para gerenciar e avaliar disponibilidade, penalidade, custo, receita e lucro. Além de métricas específicas relacionadas à qualidade do serviço de cada fatia.

Para validar tal solução, optou-se por modelar uma arquitetura baseada nas propriedades do projeto Hyperledger [Androulaki et al. 2018]. Hyperledger é uma arquitetura modular de cadeia de blocos que permite a conexão de componentes tais como mecanismo de consenso, serviços de membros e funções de transação. Esta característica permite a personalização efetiva da plataforma, conforme as necessidades do ambiente. Para representar o cenário de fatiamento de rede, optou-se por realizar um estudo de caso com a plataforma NECOS [Maciel et al. 2019].

O consenso pode ser obtido de diferentes maneiras, seja com o uso de algoritmos de loteria, como Proof of Elapsed Time (PoET) e Proof of Work (PoW), ou através do uso de métodos baseados em votação, tais como Practical Byzantine Fault Tolerance (PBFT) e RAFT. O método de consenso definido inicialmente foi o RAFT, pois é um método baseado em um mecanismo que busca o consenso através da eleição de um líder, responsável pelo gerenciamento do consenso envolvendo as transações [Ongaro and Ousterhout 2014].

O modelo proposto permite que as organizações participem de múltiplas redes blockchain independentes, através dos canais representados pelos círculos na Figura 2. Toda transação deve ser executada em um canal, no qual todos os participantes devem ser autenticados e autorizados a realizar transações. O canal oferece o compartilhamento de uma infraestrutura, mantendo a privacidade dos dados e da comunicação, e é composto pelos nodos (peer) pertencentes às organizações participantes, nodos âncora por membro (anchor peer), nodos de ordenamento das transações (order peer), livro razão compartilhadoshared ledger e contratos inteligenteschaincodes.

A implementação proposta neste trabalho consiste em uma blockchain composta por 3 organizações, conduzindo transações privadas e confidenciais através dos canais, que possuem três nodos (peer) cada, sendo um deles, um serviço de ordenação das 
transações (oderer peer), uma Certified Authority (CA) e contratos inteligentes representados em azul escuro e vermelho na Figura 2. Cada transação na rede é executada em um canal, onde cada parte deve ser autenticada e autorizada a realizar transações naquele canal. Cada membro que se junta a um canal tem sua própria identidade, fornecida por um provedor de serviços de associação (Membership Services Provider - MSP), que autentica cada membro em seus nodos de canal e serviços.

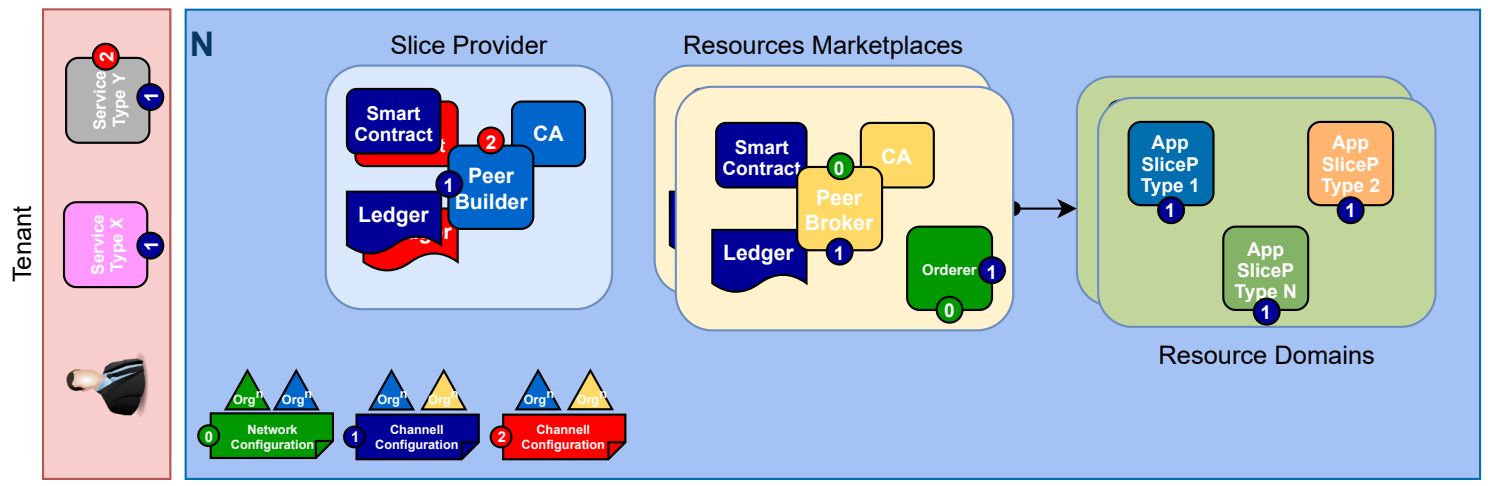

Figura 2. Modelo de arquitetura proposta para solução autônoma de monitoramento de SLA baseada no Hyperledger Fabric

Para criar um novo canal (adicionar um novo mercado de recursos), o SDK do cliente chama o sistema de configuração do canal (channel configuration) e faz referência a propriedades, como pares âncora e membros (organizações). Esta solicitação cria um bloco gênesis (genesis block) para o ledger do novo canal, que armazena informações de configuração sobre as políticas do canal, membros e nodos âncora.

Embora um nodo âncora possa pertencer a vários canais e, portanto, manter vários livros-razão, nenhum dado do livro-razão pode passar de um canal para outro. Esta separação de livros-razão, por canal, é definida e implementada pelo sistema de configuração do canal (channel configuration).

A plataforma também dá suporte a uma variedade de sistemas de gerenciamento de banco de dados, e permite a configuração das políticas de endosso e validação exigidas. No Hyperledger Fabric, a blockchain é composta por nodos pertencentes às organizações que compõem o consórcio, além de um ou mais nodos do tipo orderer, responsáveis por ordenar as transações. Para poderem se comunicar, estes nodos devem participar de um mesmo canal [Miers et al. 2020].

Os nodos do banco de dados são responsáveis pelo armazenamento do ledger contendo as transações validadas. É importante notar que todo nodo peer demanda um nodo de banco de dados, que será responsável pelo armazenamento do ledger.

Para realizar a execução e interação com os chaincodes, a proposta irá usar transações. Conforme apresenta Dhillon et al (2017) existem dois tipos de transações: Invoke e Query. A transação do tipo invoke executa um chaincode na blockchain, enquanto a transação query executa uma consulta. Através de uma transação invoke é possível a um cliente executar uma função específica contida em um chaincode. A Figura 3 ilustra como aplicações interagem com os nodos para acessar o ledger

Neste exemplo, a aplicação Service Type Y, um exemplo de fatia de locatário, co- 
necta ao nodo Peer Broker, no componente resource marketplace e realiza um invoke do chaincode (setas 1 e 2) para realizar uma atualização do ledger. O nodo Peer Broker invoca seu contrato (seta 2.1), em azul escuro, solicitando uma resposta contendo o resultado da solicitação de atualização do locatário (seta 2.2) e retorna a resposta para a aplicação (seta 3).

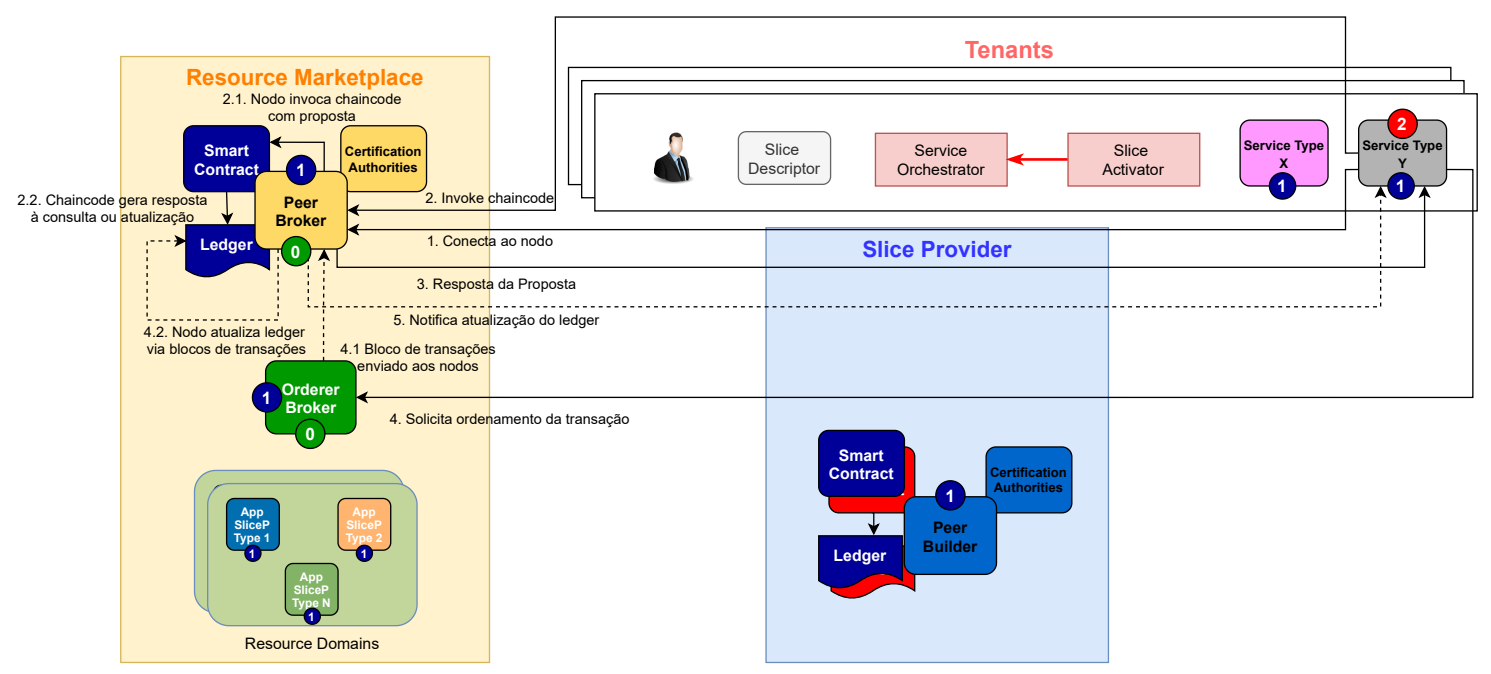

Figura 3. Fluxo de transações da proposta no Hyperledger Fabric

A partir disso, a aplicação monta uma transação contendo todas as respostas e envia ao ordenador (seta 4). Caso a solicitação fosse apenas uma consulta ao ledger, o processo estaria completo. Em seguida, o ordenador coleta as transações e as agrupa em blocos (seta 4.1), distribuindo-os a todos os nodos da rede para fornecer as informações de desempenho de cada App Agent que está sobre responsabilidade do broker. O Peer Broker valida a transação antes de adicioná-la ao ledger (seta 4.2). Por fim, Peer Broker gera um evento e envia à aplicação (seta 5).

\section{Caso de Uso: Projeto NECOS}

A plataforma NECOS não lida com os aspectos legais e econômicos relacionados à violação do SLA, ou seja, não considera tais aspectos no monitoramento proposto pelo SLO-ML que é usado no subsistema do Service Resource Orchestrator. Esta seção irá abordar como os acordos de nível de serviço entre os locatários e os provedores de recursos podem ser otimizados através da tecnologia de contratos inteligentes por meio de uma solução autônoma da aplicação.

No projeto NECOS espera-se que a organização que faz parte do Slice Provider tenha parceria com um ou mais mercados de fatia (resource marketplace). No Slice Provider do NECOS, podem ser inseridos dois nodos que correspondem a organização do Slicing Orchestrator. No modelo proposto, ilustrado na Figura 4, as conexões entre esses mercados são representadas pelos canais (vermelho e azul escuro). De semelhante modo, um marketplace pode fazer parte de um ou mais provedores de recursos (slice provider)

Um resource marketplace tem o papel de realizar o gerenciamento de uma quantidade específica de slice agents que podem ser definidos como: dc-slice e net-slice. A Figura 4 mostra a relação entre agentes representadas através de Applications Programming Interface (APIs). Dessa forma, para cada tipo de fatia é definida uma API semelhante. 
$\mathrm{Na}$ Figura 4, observa-se como será realizado o processo de auditoria e monitoramento das transações na rede. Com a utilização do serviço de eventos baseados nos canais (ChannelEvent) uma API cliente pode usar o nodo para registrar um "ouvinte"para receber blocos à medida que são adicionados ao ledger do canal. Dessa forma um locatário pode processar os blocos de entrada e procurar transações específicas ou eventos chaincode. Isso permite que uma API cliente possa ser notificada sobre a conclusão da transação ou eventos de chaincode arbitrários sem ter que realizar várias consultas ou pesquisar os blocos à medida que são recebidos. A informação é enviada para o banco de dados e a partir desse ponto a rede blockchain irá ter como responsabilidade monitorar os recursos instanciados de modo a prevenir violações contratuais de SLA.

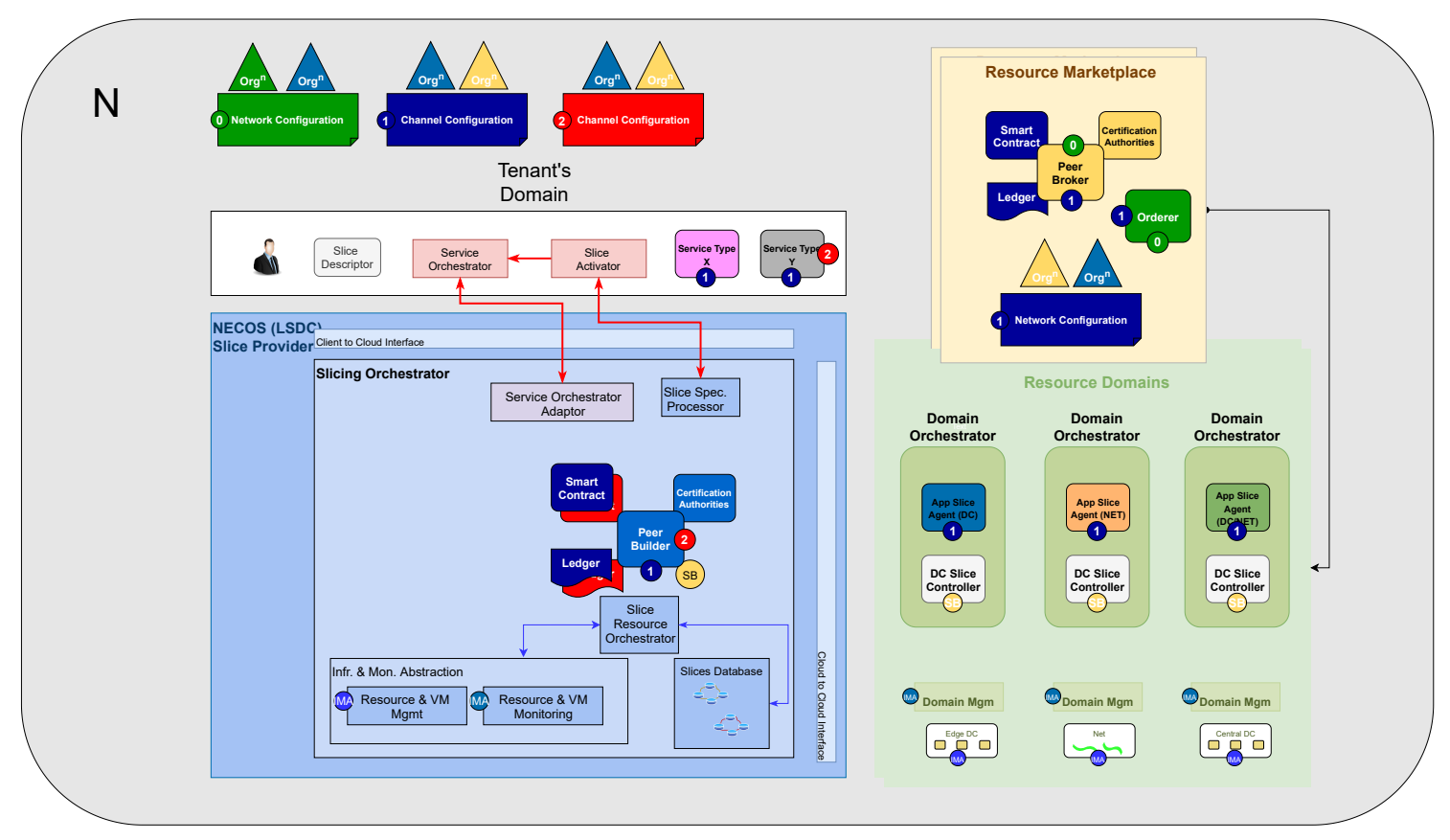

Figura 4. Modelo de arquitetura aplicada no cenário NECOS

O Slicing Orchestrator é um componente de suma importância na arquitetura do NECOS, pois, além de ser responsável pela orquestração dos recursos, ele também é responsável pela instanciação dos VIMs/WIMs. A instanciação destes elementos é de suma importância para o deployment de um novo serviço e/ou recurso virtual (Máquina Virtual, Container). Para isto, ele se comunica diretamente com os DC/WAN Slice Controllers.

Na proposta deste trabalho é incluído neste componente o processo de consulta de métricas de SLA através de Chaincode Events. Dessa forma o DC WAN Slice Controller que se comunica diretamente com o Peer Builder retorna a requisição da operação que é gerenciada pelo Slice Provider (especificamente o Slicing Orchestrator).

Por exemplo, um chaincode pode definir um conjunto de métricas de violações de SLA e suas penalidades subsequentes, e através dos channel events é possível realizar o monitoramento de cada transação registrada no livro razão em tempo real, portanto, pode-se prover uma solução autônoma de monitoramento e auditoria desses acordos de nível de serviço para o processo de fatiamento de recursos voltados para um comércio de multi-mercado. 
A Figura 5 apresenta um fluxo que foi adaptado para exemplificar a proposta. Ela utiliza a metodologia BPMN (do inglês, Business Process Model and Notation), que relaciona os componentes presentes na arquitetura proposta na Figura 4 . O fluxo foi adaptado e é detalhado no Entregável D5.1 do NECOS [NECOS 2018], que envolve a operação de criação de uma nova fatia.

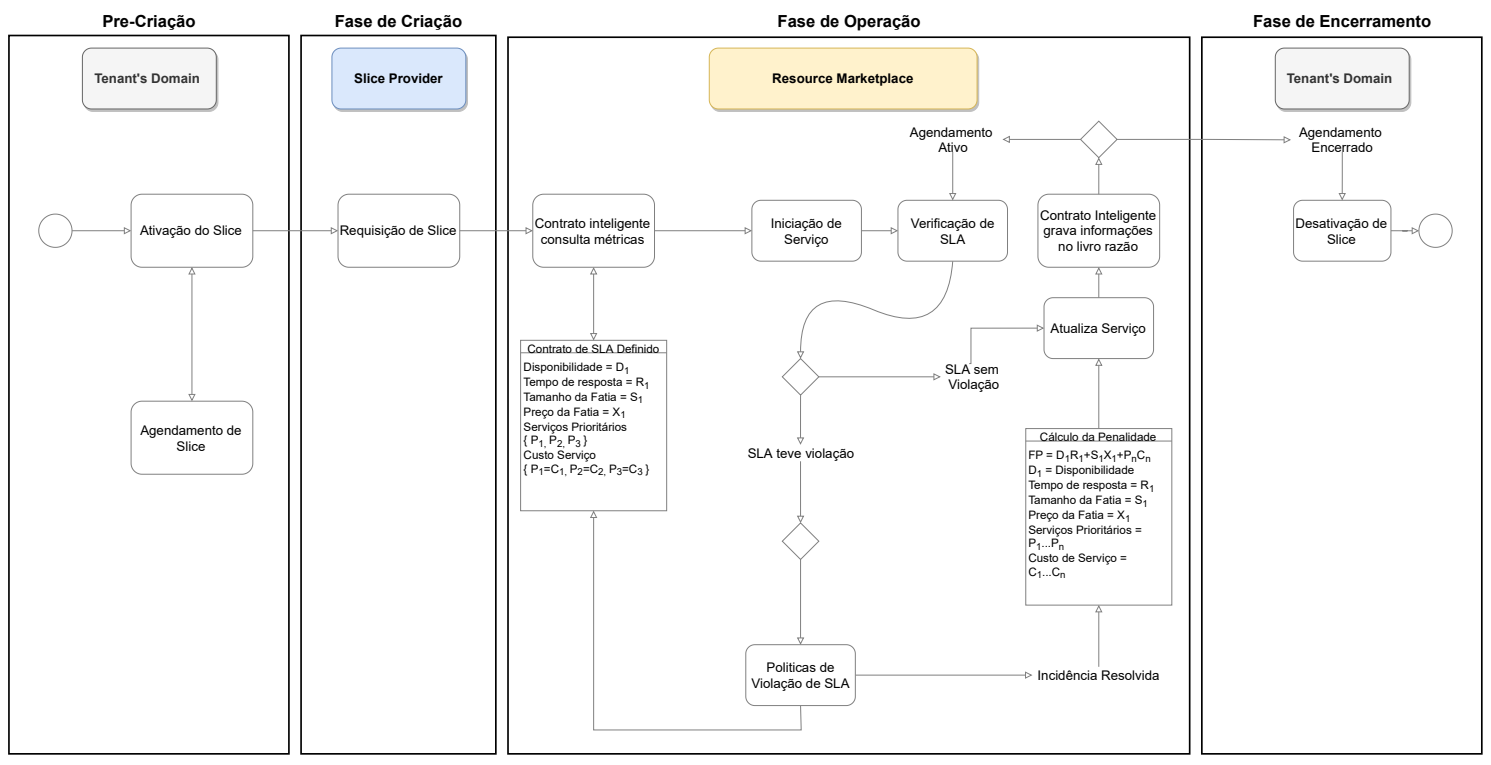

Figura 5. Fluxo de Monitoramento do Ciclo de Vida da Fatia

O fluxo adota a seguinte notação: os círculos denotam o início e o término do fluxo. As setas representam as interações entre os processos nos componentes. Os retângulos coloridos representam os componentes da arquitetura do NECOS. Os retângulos sem coloração representam processos dentro de cada componente, os losangos indicam condições que ocorrem em decorrência dos processos nos componentes. Por fim, os scripts de contratos nos processos entre os componentes são ilustradas pelas entidades representadas pelas tabelas.

O fluxo começa com locatário através do processo "Ativação de Slice", a sua função é iniciar a requisição de uma fatia para o componente Slice Provider. Após a "Requisição de Slice"o Slice Provider analisa os recursos disponíveis nos provedores de infraestrutura e então otimiza a alocação dos recursos ofertados pelos multi-mercados. No Resouce Marketplace o processo “Contrato Inteligente Consulta Métricas"é chamado para iniciar o ciclo de serviços.

O ciclo no componente Resource Marketplace consiste na criação de um contrato inteligente com métricas pré-definidas por cada provedor de recurso para a fatia contratada pelo locatário. A Figura 5 apresenta um exemplo de um contrato simples de SLA com métricas de penalidades básicas. Após cada atualização do ledger, o processo de "Verificação de SLA"é iniciado para monitorar o desempenho dos provedores de recursos. Após o período de agendamento de consumo da fatia ser esgotado o processo de "Desativação de Slice"no domínio do locatário encerra o ciclo.

A tecnologia DLT que é embutida nos projetos de tecnologia blockchain pode trazer confiança ao processo de manutenção de registros no monitoramento e gerenciamento 
de SLA. Em outras palavras, as medições dos parâmetros de qualidade com os incidentes de violação de SLA podem ser mantidas como registros imutáveis no livro razão distribuído. A rede Blockchain pode ser configurada para ser um agente intermediário que irá gerenciar (através de orderers) contratos inteligentes para estabelecer confiança entre os contratos de nível de serviço entre os provedores de recursos da plataforma.

\section{Conclusões e Trabalhos Futuros}

Os contratos inteligentes têm a proposta de serem facilmente programáveis para se adaptarem a qualquer modelo de negócio, sendo uma solução para muitas organizações que ainda realizam o processo de monitoramento de SLA de forma manual. Com a integração da blockchain no comércio de fatia entre diferentes domínios administrativos, é possível estabelecer parcerias através de contratos inteligentes projetados para rastrear a negociação, monitorar perfis de instância de fatia e acionar eventos em violações de contrato. Através do modelo de gerenciamento e monitoramento de recursos e serviços de fatias de rede será possível garantir a integridade de informações de fluxo.

Como trabalhos futuros, pretende-se realizar a implementação desta proposta de rede blockchain e configuração dos chaincodes para incluir na arquitetura do projeto NECOS. A definição de um contrato inteligente capaz de gerenciar o monitoramento dos provedores de recursos e serviços das fatias, permite uma visualização em tempo real dos dados que são registrados na rede. Estima-se obter resultados de testes com o modelo para replicar para estudos de caso dentro do escopo.

Para uma avaliação do desempenho da rede, a primeira etapa será avaliar e identificar a granularidade dos dados necessária para realizar o monitoramento dos $\log s$ sem prejudicar o desempenho da rede. É fundamental identificar os dados a serem monitorados, visto que todo registro de dados em uma rede blockchain é lento, devido à sua duplicabilidade.

\section{Agradecimentos}

O presente trabalho foi realizado com apoio da Coordenação de Aperfeiçoamento de Pessoal de Nível Superior - Brasil (CAPES) - Código de Financiamento 001.

\section{Referências}

Afraz, N. and Ruffini, M. (2020). 5g network slice brokering: A distributed blockchainbased market. In 2020 European Conference on Networks and Communications $(E u C N C)$, pages 23-27.

Androulaki, E., Barger, A., Bortnikov, V., Cachin, C., Christidis, K., De Caro, A., Enyeart, D., Ferris, C., Laventman, G., Manevich, Y., and et al. (2018). Hyperledger fabric. Proceedings of the Thirteenth EuroSys Conference.

Backman, J., Yrjölä, S., Valtanen, K., and Mämmelä, O. (2017). Blockchain network slice broker in $5 \mathrm{~g}$ : Slice leasing in factory of the future use case. In 2017 Internet of Things Business Models, Users, and Networks, pages 1-8.

Buterin, V. et al. (2014). A next-generation smart contract and decentralized application platform. white paper, 3(37). 
Clayman, S., Tusa, F., and Galis, A. (2018). Extending slices into data centers: the vim on-demand model. In 2018 9th International Conference on the Network of the Future (NOF), pages 31-38.

Dhillon, V., Metcalf, D., and Hooper, M. (2017). The hyperledger project. In Blockchain Enabled Applications, page 139-149. Springer.

Elhabbash, A., Elkhatib, Y., Blair, G., Lin, Y., and Barker, A. (2019). A framework for slo-driven cloud specification and brokerage. In 2019 19th IEEE/ACM International Symposium on Cluster, Cloud and Grid Computing (CCGRID), pages 666-667.

Farias, F., Pinheiro, B., Abelém, A., Jr, P. M., Rocha, A., Verdi, F., Neto, A., Correa, S., Nascimento, M., Pasquini, R., and Rothenberg, C. (2019). Projeto necos: Rumo ao fatiamento leve de recursos em infraestruturas de nuvens federadas. In Anais do X Workshop de Pesquisa Experimental da Internet do Futuro, pages 56-63, Porto Alegre, RS, Brasil. SBC.

Habibi, M. A., Han, B., Nasimi, M., and Schotten, H. D. (2018). The structure of service level agreement of slice-based $5 \mathrm{~g}$ network.

Kumar, M., Dubey, K., and Pandey, R. (2021). Evolution of emerging computing paradigm cloud to fog: Applications, limitations and research challenges. In 2021 11th International Conference on Cloud Computing, Data Science Engineering (Confluence), pages $257-261$.

Lieto, A., Malanchini, I., and Capone, A. (2018). Enabling dynamic resource sharing for slice customization in 5g networks. In 2018 IEEE Global Communications Conference (GLOBECOM), pages 1-7.

Maciel, P. D., Verdi, F. L., Valsamas, P., Sakellariou, I., Mamatas, L., Petridou, S., Papadimitriou, P., Moura, D., Swapna, A. I., Pinheiro, B., and Clayman, S. (2019). A marketplace-based approach to cloud network slice composition across multiple domains. In 2019 IEEE Conference on Network Softwarization (NetSoft), pages 480-488.

Miers, C. C., Koslovski, G. P., Pillon, M. A., and Marques, M. A. (2020). Blockchains com hyperledger: conceitos, instalação, configuração e uso. Sociedade Brasileira de Computação.

NECOS (2018). D5.1: Architectural update, Monitoring and Control Policies Frameworks.

NECOS (2019). D3.2: NECOS System Architecture and Platform Specification. V2.

Ongaro, D. and Ousterhout, J. (2014). In search of an understandable consensus algorithm. In 2014 USENIX Annual Technical Conference (USENIX ATC 14), pages 305319, Philadelphia, PA. USENIX Association.

Rathi, V. K., Chaudhary, V., Rajput, N. K., Ahuja, B., Jaiswal, A. K., Gupta, D., Elhoseny, M., and Hammoudeh, M. (2020). A blockchain-enabled multi domain edge computing orchestrator. IEEE Internet of Things Magazine, 3(2):30-36.

Rebello, G., Camilo, G., Silva, L., Guimaraes, L., Souza, L., Alvarenga, I., and M. B. Duarte, O. C. (2019). Providing a sliced, secure, and isolated software infrastructure of virtual functions through blockchain technology. In 2019 IEEE 20th International Conference on High Performance Switching and Routing (HPSR), pages 1-6. 
Reyna, A., Martín, C., Chen, J., Soler, E., and Díaz, M. (2018). On blockchain and its integration with iot. challenges and opportunities. Future Generation Computer Systems, 88:173-190.

Rosa, R. V. and Rothenberg, C. E. (2018). Blockchain-based decentralized applications for multiple administrative domain networking. IEEE Communications Standards Magazine, 2(3):29-37.

Sciancalepore, V., Costa-Perez, X., and Banchs, A. (2019). Rl-nsb: Reinforcement learning-based $5 \mathrm{~g}$ network slice broker. IEEE/ACM Transactions on Networking, 27(4):1543-1557.

Seitz, A., Henze, D., Miehle, D., Bruegge, B., Nickles, J., and Sauer, M. (2018). Fog computing as enabler for blockchain-based iiot app marketplaces - a case study. In 2018 Fifth International Conference on Internet of Things: Systems, Management and Security, pages 182-188.

Silva, F. S. D., Lemos, M. O. O., Medeiros, A., Neto, A. V., Pasquini, R., Moura, D., Rothenberg, C., Mamatas, L., Correa, S. L., Cardoso, K. V., Marcondes, C., ABelem, A., Nascimento, M., Galis, A., Contreras, L., Serrat, J., and Papadimitriou, P. (2018). Necos project: Towards lightweight slicing of cloud federated infrastructures. In 2018 4th IEEE Conference on Network Softwarization and Workshops (NetSoft), pages 406414.

Soenen, T., Banerjee, R., Tavernier, W., Colle, D., and Pickavet, M. (2017). Demystifying network slicing: From theory to practice. In 2017 IFIP/IEEE Symposium on Integrated Network and Service Management (IM), pages 1115-1120.

Swapna, A. I., Rosa, R. V., Rothenberg, C. E., Sakellariou, I., Mamatas, L., and Papadimitriou, P. (2019). Towards a marketplace for multi-domain cloud network slicing: Use cases. In 2019 ACM/IEEE Symposium on Architectures for Networking and Communications Systems (ANCS), pages 1-4.

Valtanen, K., Backman, J., and Yrjölä, S. (2018). Creating value through blockchain powered resource configurations: Analysis of 5g network slice brokering case. In 2018 IEEE Wireless Communications and Networking Conference Workshops (WCNCW), pages $185-190$. 